\title{
PHENOTYPICAL AND MASS SPECTRAL ASSESSMENT METHODS FOR IDENTIFICATION OF SOME CONTAGIOUS MASTITIS PATHOGENS
}

\author{
${ }^{1,3}$ El Behiry, A., ${ }^{1,5}$ Rasha Nabil Zahran, \\ ${ }^{2,4}$ Eman Marzouk and ${ }^{3}$ Musaad Al-Dabib \\ ${ }^{1}$ Department of Bacteriology, Mycology and Immunology, \\ Faculty of Veterinary Medicine, University of Sadat City, Sadat City, Egypt \\ ${ }^{2}$ Veterinary Medicine Directorate-El Beheira, Ministry of Agriculture, Egypt \\ ${ }^{3}$ Department of Public Health, Faculty of Public Health, Qassim University, Qassim Region, KSA \\ ${ }^{4}$ Department of Scientific Unit (Biology), Deanship of Educational Services, QassimUniversity, KSA \\ ${ }^{5}$ Department of Medical Microbiology, Faculty of Applied Medical Science, Taraba, Taif University, KSA
}

Received 2014-04-12; Revised 2014-04-19; Accepted 2014-05-29

\begin{abstract}
Mastitis is one of the most economic disease affecting dairy cows worldwide. Identification of mastitis pathogens still depends principally on culture and phenotypical method, which is a difficult and timeconsuming. Newly, microbiologists have focused their attention on the use of Mass Spectrometry (MS) for microbial identification, especially Matrix Assisted Laser Desorption Ionization Time-Of-Flight (MALDITOF). Therefore, this study was designated to evaluate the ability of MALDI-TOF to identify some contagious mastitis pathogens comparing with phenotypical methods such as API panels and VITEK 2 system. A total of one hundred twenty of Staphylococcus aureus (S. aureus), Coagulase Negative Staphylococci (CNS) and Streptococcus agalactiae (Strept. agalactiae) strains isolated from milk of cows affected by clinical and subclinical mastitis were used in the study. According to the results, $\sim 95 \%$ of S. aureus, $100 \%$ of CNS and Strept. agalactiae were correctly identified by MALDI TOF MS. All S. aureus isolates were then confirmed by a nuc-based PCR technique. While $~ 92 \%$ of S. aureus, $87 \%$ of Strept. agalactiae and $76 \%$ of CNS were identified by VITEK 2 system. Moreover, $~ 89 \%$ of S. aureus, $80 \%$ of Strept. agalactiae and $72 \%$ of CNS were identified by API system. In brief, the results demonstrated that MALDI-TOF is a fast and truthful technique which has the capability to replace conventional identification of several bacterial strains usually isolated in clinical laboratories of microbiology. Therefore, it is recomended that MALDI-TOF MS technology can be regularly used in veterinary laboratories for identification of different species of bacteria, particularly when failure of phenotypic methods forces clinical microbiologists.
\end{abstract}

Keywords: Phenotype, MALDI-TOF-MS, Identification, Mastitis Pathogens

\section{INTRODUCTION}

Mastitis represents one of the most significant problems in modern dairy production from the economic, diagnostic and public-health related point of view (Benić et al., 2012). About 150 species of microorganisms, mostly bacteria is able to cause mastitis (Dudko et al., 2010). They are divided in two groups: Contagious and environmental udder pathogens. The most common contagious pathogens causing mastitis in dairy cattle worldwide and $50-100 \%$ of herds may be infected with these pathogens are S. aureus and Streptococcus agalactiae (Strept.

Corresponding Author: El Behiry, A., Department of Bacteriology, Mycology and Immunology, Faculty of Veterinary Medicine, University of Sadat City, Egypt Tel: 00966532207969 
agalactiae) (Giebel et al., 2010). These pathogens have adapted to survive within the mammary gland and are distribute among cows nearly at the time of milking (Pradhan et al., 2011). Although the group of contagious pathogens is small regarding the number of species included, in many countries this group is more often isolated from udder secretions (Benić et al., 2012). The majority of intra-mammary infections due to $S$. aureus and Strep. agalactiae are subclinical and hence the response of this infection to treatment is comparatively poor and it causes premature culling and elimination of animals from herd (Saidi et al., 2013). Furthermore, the presence of $S$. aureus in milk may a public health problem to the consumer because of the ability of this type of bacteria to produce enzymes and enterotoxins which causes serious food poisoning (Johler et al., 2013).

Concerning the importance of $S$. aureus and Strept. agalactiae, examining of flock for diagnosis of the initial cases of infection is necessary for prevention of infection spreading. Although the culture of milk is considered as a gold standard test for mastitis diagnosis, there are several disadvantages associated with bacterial culture, including no bacterial yielding from truly subclinically infected cows due to presence of high number of leukocytes or presence of preservatives or residual therapeutic antibiotics in submitted samples. A further recognized disadvantage of culture is that about 25 to $45 \%$ of all milk samples yield no bacterial growth even after two days of incubation (Koskinen et al., 2010). Moreover, microbiological culture of milk is time consuming and species identification by standard biochemical methods requires more than $48 \mathrm{~h}$ to be completed. Due to the above-mentioned limitations of cultural methods, Polymerase Chain Reaction (PCR) has been developed to identify various mastitis pathogens (Koskinen et al., 2010). The progress of PCR based techniques supplies a talented option for the rapid detection of different microorganisms. Using of this technique, most of bacterial species can be identified in hours, instead of days needed for classical culture methods. PCR has high sensitivity and specificity and can improve the level of detection (Boss et al., 2011; Castelani et al., 2013).

Both biochemical and genetic analyses are truthful, but their utilize may be prolonged and their cost comparatively high. Therefore, there is a still require for additional fast and inexpensive but precise techniques of identifying microorganisms of various communicable diseases. Overall, novel skills for particular and rapid identification of microorganisms are a very important step toward a appropriate treatment of infectious diseases in veterinary and medical diagnostics and are of major concern today (Hays and Leeuwen, 2012). Identification of different pathogens with mass spectrometry has been known as a technique for acurate and quick recognation of bacteria (Giebel et al., 2010; Karger et al., 2012). Consequently, the most commonly applied technique for mass spectral identification of microorganisms is Matrix Assisted Laser Desorption Ionization Time-Of-Flight Mass Spectrometry (MALDI-TOF-MS) (Biswas and Rolain, 2013). Mass spectral identification of various microorganisms by MALDI-TOF-MS can be suitable to a huge types of bacteria and fungi. In recent times, mass spectral identification of bacteria has been investigated using MALDI-TOF MS (Barreiro et al., 2010). Many microorganisms have been identified by MALDITOF MS via characteristic "chemical signatures" in a highthroughput mode using simple extraction and sample work-up protocols. After initial bacterial growth that requires one day on average and using crude bacterial extracts or lysate supernatants of whole cells, bacterial determination has been performed by MALDI-TOF MS in a few minutes (Alispahic et al., 2010; Barreiro et al., 2010; El-Bouri et al., 2012). This technique has therefore improved the field of bacterial screening by providing a much faster, more reliable, cheaper and highly sensitive technique for bacterial identification. These features are particularly attractive for bacterial isolates screening in contaminated milk at subclinical levels (Barreiro et al., 2010; El-Bouri et al., 2012). In addition, identification of bacteria by MALDI-TOF-MS has revealed prominent interlaboratory reproducibility (Mellmann et al., 2009) and is quickly being included as a routine method for animal and human laboratory microbiology. This technique requires slight Mass Spectral (MS) ability, the utilize of uncomplicated instruments and relatively fast operator training. After comparing mass spectral identification with DNA analysis based technologies, it was found that MALDI-TOF MS requires smaller amounts of biological material and involves simpler sample preparation protocols with no initial assessment, such as Gram staining (Ilina et al., 2009; 2010; Chalupová et al., 2014; Tomazia et al., 2014). In the current study, the performance of MALDI-TOF-MS was evaluated comparing with conventional methods for bacterial screening of clinical and subclinical mastitis in dairy cows.

\section{MATERIALS AND METHODS}

\subsection{Samples}

Over a 12-weeks period milk samples were collected from four dairy farms with high incidence of contagious mastitis pathogens (S. aureus, Strept. agalactiae) in ElBehira and Alexandria Governorates, Egypt and Al- 
Qassim region, Kingdom of Saudi Arabia, using standard measures explained by the National Mastitis Council. From every herd, fifty cows in various phases of lactation and dissimilar age groups were chosen for sampling. Prior to collection of samples; teat ends were spotless with warm water and dried before 10 to $15 \mathrm{~mL}$ of milk was drawn and discarded. The teat ends were then scrubbed with cotton or paper towel containing $70 \%$ ethanol and then the milk samples were transferred on ice-covered and kept at $-20^{\circ} \mathrm{C}$ until analysis.

\subsection{Bacterial Isolation}

About 120 isolates of bacteria isolated from samples of milk were collected from the four dairy herds. Isolation of all isolates was carried out according to the National Mastitis Council suggestions on assessment of mastitic cows milk. The isolates were stored at $-80^{\circ} \mathrm{C}$ for further investigation. To isolate bacteria from milk samples, $10 \mu \mathrm{L}$ of sampled milk was inoculated onto blood agar plates and incubated at $37^{\circ} \mathrm{C}$ for one day to permit of bacterial growth.

\subsection{Classical Identification}

Standard identification of different pathogens was carried out by a variety of phenotypic tests, as the coagulase test (Becton, Dickinson and Company, Sparks, Maryland, USA), the catalase test (bioMe'rieux, Marcy l'Etoile, France), tests with the API and VITEK 2 Systems (bioMérieux). Quality control of commercial systems was carried out by periodically testing a range of ATCC strains. The criteria used for the approval of the identifications obtained with the API and VITEK 2 systems were that the isolate was identified with $>90 \%$ probability.

\subsection{Identification with API System}

The API system strip (BioMe'rieux, Paris, France) consists of 32 cupules, 26 of which contain dehydrated biochemical agents for colorimetric examinations. The tests included acid production from Urea (URE), Larginine (ADH), Lornithine (ODC), Esculin (ESC), Dglucose (GLU), D-fructose (FRU), D-mannose (MNE), Dmaltose (MAL), D-lactose (LAC), D-trehalose (TRE), D-mannitol (MAN), D-Raffinose (RAF), D-Ribose (RIB), D-cellobiose (CEL), potassium Nitrate (NIT), sodium pyruvate (VP), 2-naphthy- BDGalactopyranoside (BGAL), L-arginine B-naphthyamide (ArgA), 2-naphthyl Phosphate (PAL), pyroglutamic Acid-ß-Naphthylamide (PyrA), Novobiocin (NOVO), Sucrose (SAC), N-Acetylglucosamine (NAG), DTuranose (TUR), L-Arbianose (ARA) and 4-nitrophenylBD-Glucuronide (ßGUR). The manufacturer's recommended procedures (API System, BioMe'rieux) were followed. Briefly, 3-5 of bacterial colonies were prepared from overnight cultures on blood agar plates. They were standardized with an equivalent turbidity to 0.5 McFarland standards in $6 \mathrm{ml}$ of sterile distilled water. The ampule of inoculated API suspension medium was homogenized and $55 \mu \mathrm{L}$ of the suspension were dispensed in each cupule of the strip. The tests URE, ADH and ODC were covered with two drops of mineral oil. After an incubation period of 18 $24 \mathrm{~h}$ at $37^{\circ} \mathrm{C}$, reagents were added for the nonspontaneous tests. Strain profiles were read and identified with Automatic Testing Bacteriology (ATB) Expression and were interpreted with API Laboratory (LAB) software. This software gives the probability of the identification result in a range of 10 to $100 \%$.

\subsection{Identification with VITEK 2 System}

Identification of all isolates were detected using the automated systems Vitek2® (bioMérieux-software 4,03), panel Gram Positive (GP) card. The manufacturer's instructions were followed for the preparation of the inoculua and incubation of the isolates. The card was automatically filled by a vacuum device, sealed and inserted into the VITEK 2 reader-incubator module (incubation temperature, $35.5^{\circ} \mathrm{C}$ ) and subjected to a kinetic fluorescence measurement every $15 \mathrm{~min}$. The results were interpreted by the ID-GPC database and final results were obtained automatically. All cards used were automatically discarded into a waste container.

\subsection{MALDI-TOF MS identification}

Mass spectral identification of various strains of microorganisms using MALDITOF MS was performed on a Microflex LT device (Bruker Daltonics $\mathrm{GmbH}$, Bremen, Germany) with FlexControl (version 3.0) software (Bruker Daltonics) for the mechanical achievement of mass spectra in the linear positive mode in a range of 2 to $20 \mathrm{kDa}$, according to the instructions of the manufacturer. To prepare the samples for MALDITOF MS, cells from a single colony of fresh overnight culture (Columbia agar supplemented with $5 \%$ horse blood (bioMérieux), incubated $24 \mathrm{H}$ at $37^{\circ} \mathrm{C}$ ) were used for each isolate to prepare samples according to the microorganism profiling ethanol/acid formic extraction procedure, as recommended by the manufacturer. As can be seen from Fig. 1, the identification was first achieved by touching the surface of the investigated colony with a sterile pipette tip and directly applying the small amount of sample on polished or ground steel MSP 96 target plates (Bruker Daltonics). 


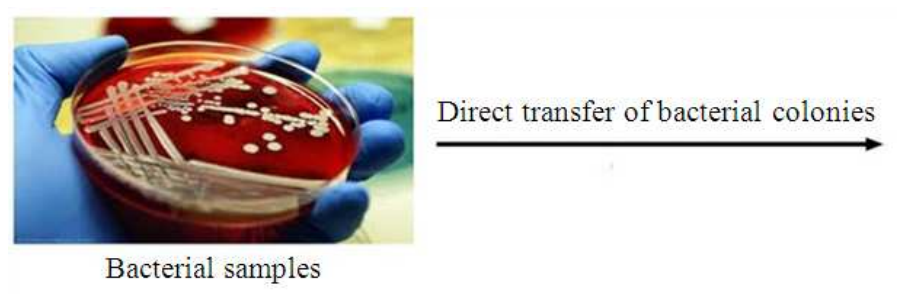

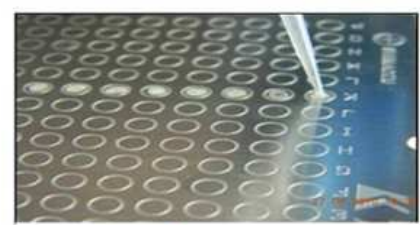

Sample preparation on MALDI target metal plate
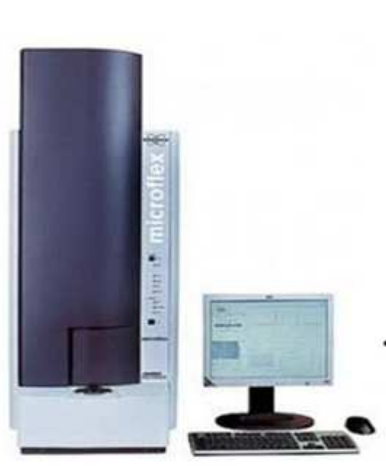

MALDI mass spectrometry
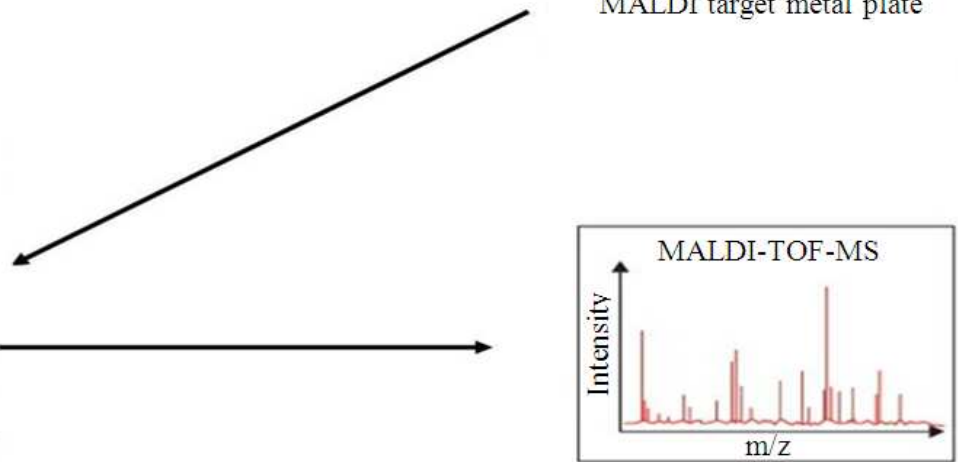

Protein mass pattern detection

Fig. 1. Steps usually used in MALDI-TOF MS for identification of bacterial isolates

The sited bacteria were enclosed with $1 \mu \mathrm{L}$ of a chemical matrix (saturated solution of $\alpha$-cyano-4-hydroxycinnamic acid in $50 \%$ acetonitrile/2.5\% trifluoroacetic acid) and air desiccated at room temperature to permit co-crystallization with the investigational sample. The spectra were then obtained by the mass spectrometer and were compared by using the BioTyper software. Each spectrum was the sum of the ions obtained from 200 laser shots performed in 5 different regions of the same well. The spectra have been analyzed in a range of 2000 to $20000 \mathrm{~m} / \mathrm{z}$. According to the measure designed by the manufacturer, a result was supposed right when the score value was $>2.0$.

\section{RESULTS}

A characteristic examination of staphylococcal and streptococcal strains isolated from clinical and subclinical cases of bovine mastitis by MALDI-TOF MS, 10 to 20 prominent ion peaks were demonstrated in the spectra from the region between 2,000 and 18,000 $\mathrm{Da}$, with the highest-intensity peaks being consistently in the range of 3,000 to $10,000 \mathrm{Da}$. On this basis, the log (score) values obtained by MALDI-TOF MS correctly identified all but two staphylococcal isolates at the species level [log (score), $\geq 2.0]$, were not identified. As can be noticed from Fig. $2 \sim 95 \%$ (37/39) of S. aureus and $100 \%(31 / 31)$ of Strep. agalactiae isolates were accurately identified by MALDI-TOF-MS. Among the five species of minor pathogens causing mastitis in cattle, $S$. xylosus $(n=11), S$. haemolyticus $(n=13), S$. epidermidis $(n=7), S$. chromogenes $(n=10)$ and $S$. saprophyticus $(n=9)$ MALDI-TOF MS gave a right identification in 100\% (50/50) (Table 1).

One hundred twenty isolates of different types of contagious mastitis pathogens were analyzed by conventional (as API ID System and VITEK 2 System) and Mass Spectral (MALDI-TOF-MS) methods as described in materials and methods. For each spectrum, a value matching to the force was given to each peak. The peak with the highest intensity was arbitrarily set to 3; all the other peaks had a value matching to the relative intensity of this highest peak (Fig. 3-5). It should be pointed out that minor peaks (relative intensity below 0.1 ) were inconstantly present. We reasoned that peaks that are species specific are likely to correspond to bacterial components created in high quantity and that such components would therefore generate conserved peaks of high relative intensity. We consequently concentrated on peaks with a relative intensity above 0.1 . In general, our data illustrate that, by selecting a suitable set of strains and keeping only the conserved peaks with an $\mathrm{m} / \mathrm{z}$ above 0.1 , a database can be engineered and used for species identification of Micrococcaceae. 
El Behiry, A. et al. / American Journal of Microbiology 5 (1): 1-10, 2014

Table 1. Mass spectral (MALDI-TOF MS) and biochemical identification of 120 bacterial strains isolated from different milk samples from cows with clinical and sub-clinical mastitis

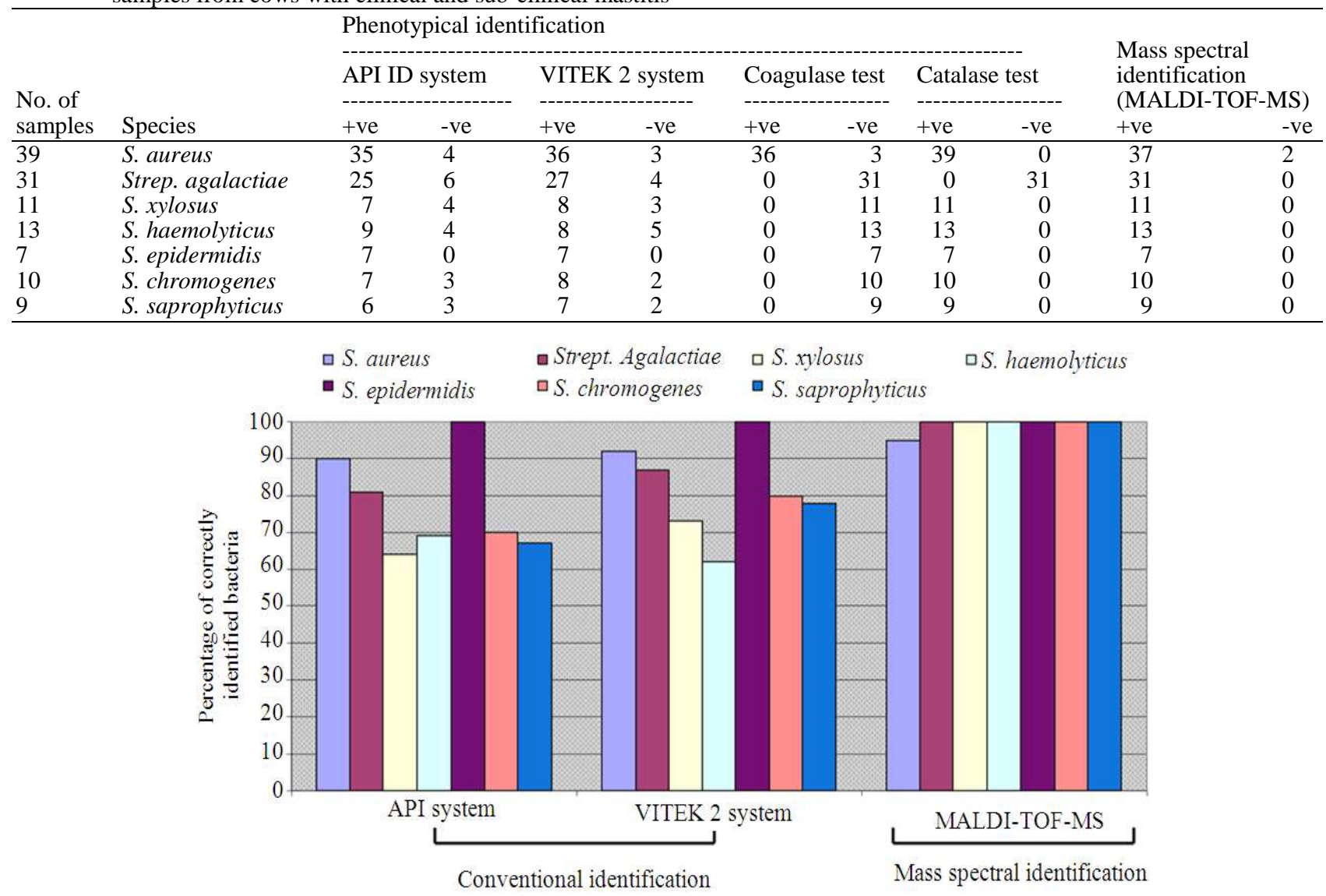

Fig. 2. Comparing the percentage of correctly identified S. aureus, Srtep. agalactiae and CNS by using conventional and mass spectral methods

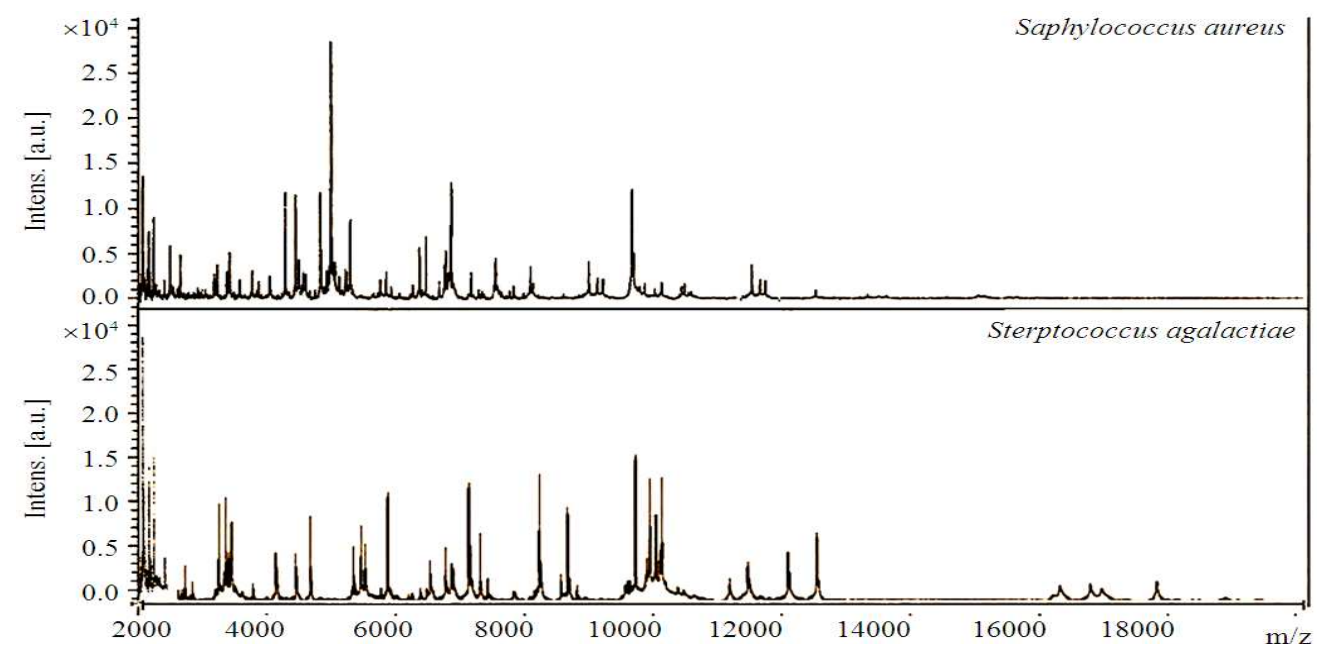

Fig. 3. MALDI-TOF-MS profiles of S. aureus and Strep. agalactiae cultured overnight at $37^{\circ} \mathrm{C}$ on Columbia blood agar plates 
El Behiry, A. et al. / American Journal of Microbiology 5 (1): 1-10, 2014

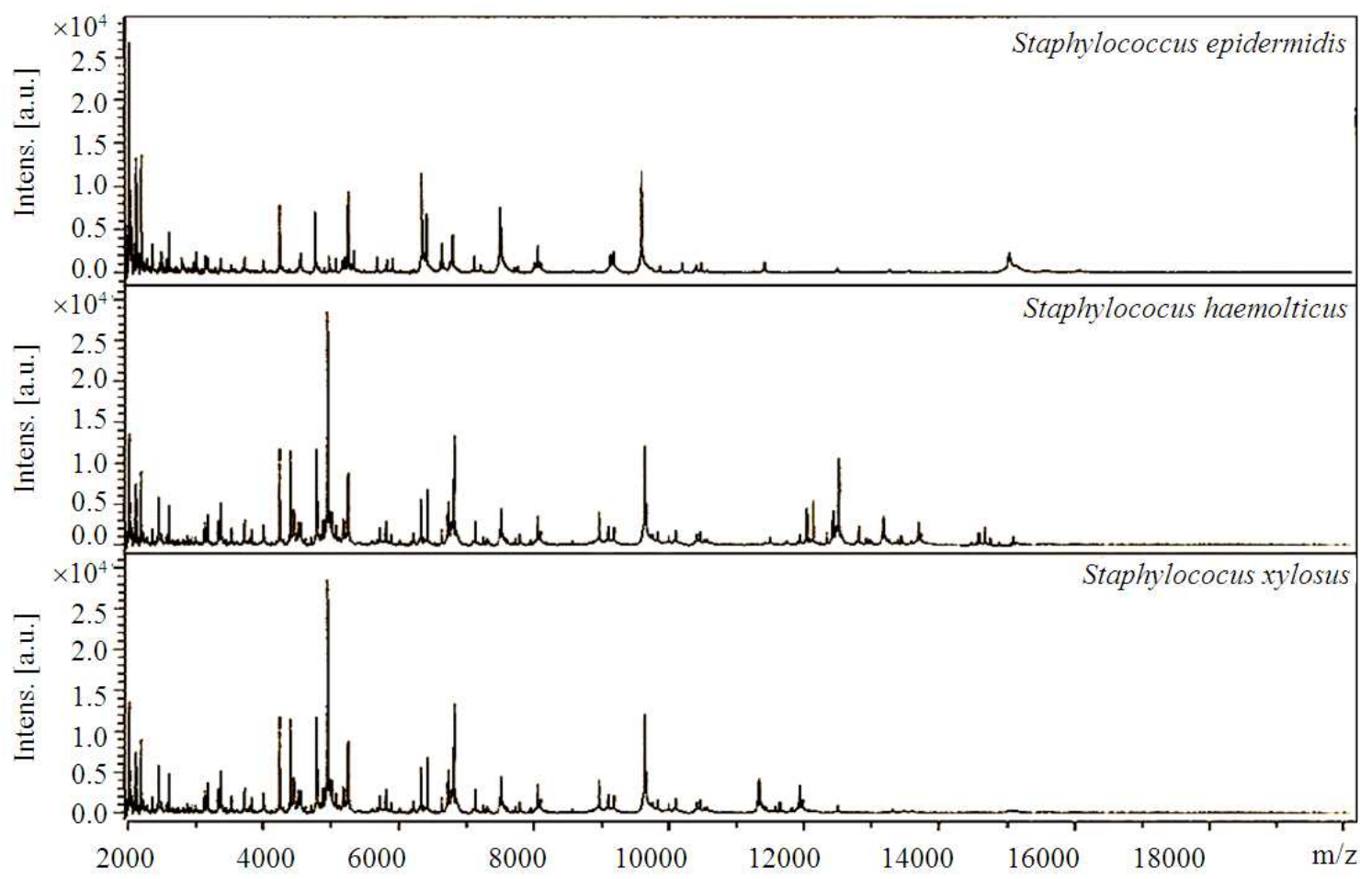

Fig. 4. MALDI-TOF-MS profiles of 3 isolates of coagulase negative staphylococci (S. epidermidis; S. haemolyticus and S. xylosus) cultured overnight at $37^{\circ} \mathrm{C}$ on Columbia blood agar plates

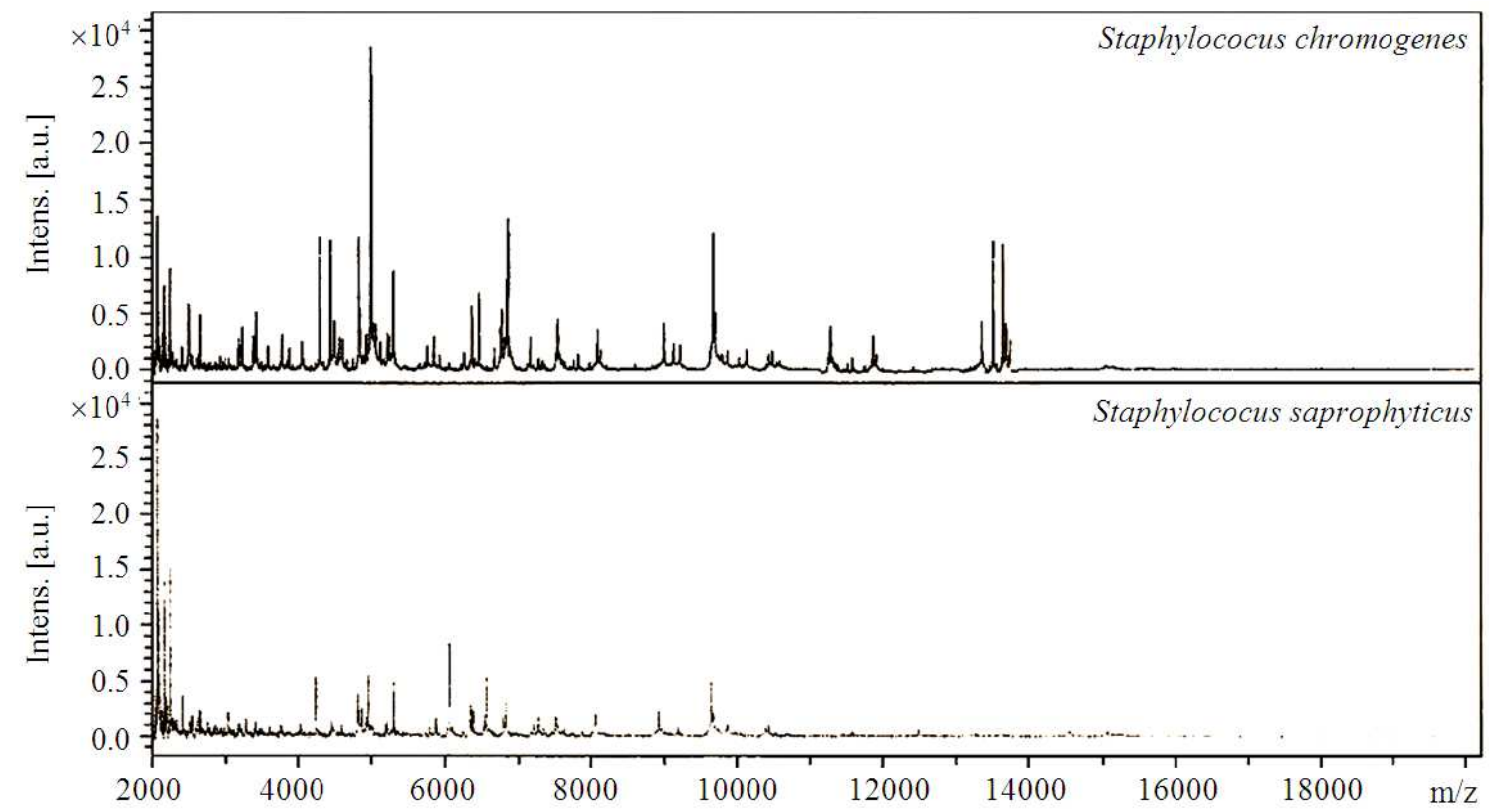

Fig. 5. MALDI-TOF-MS profiles of 2 isolates of CNS (S. chromogenes and S. saprophyticus cultured overnight at $37^{\circ} \mathrm{C}$ on Columbia blood agar plates 


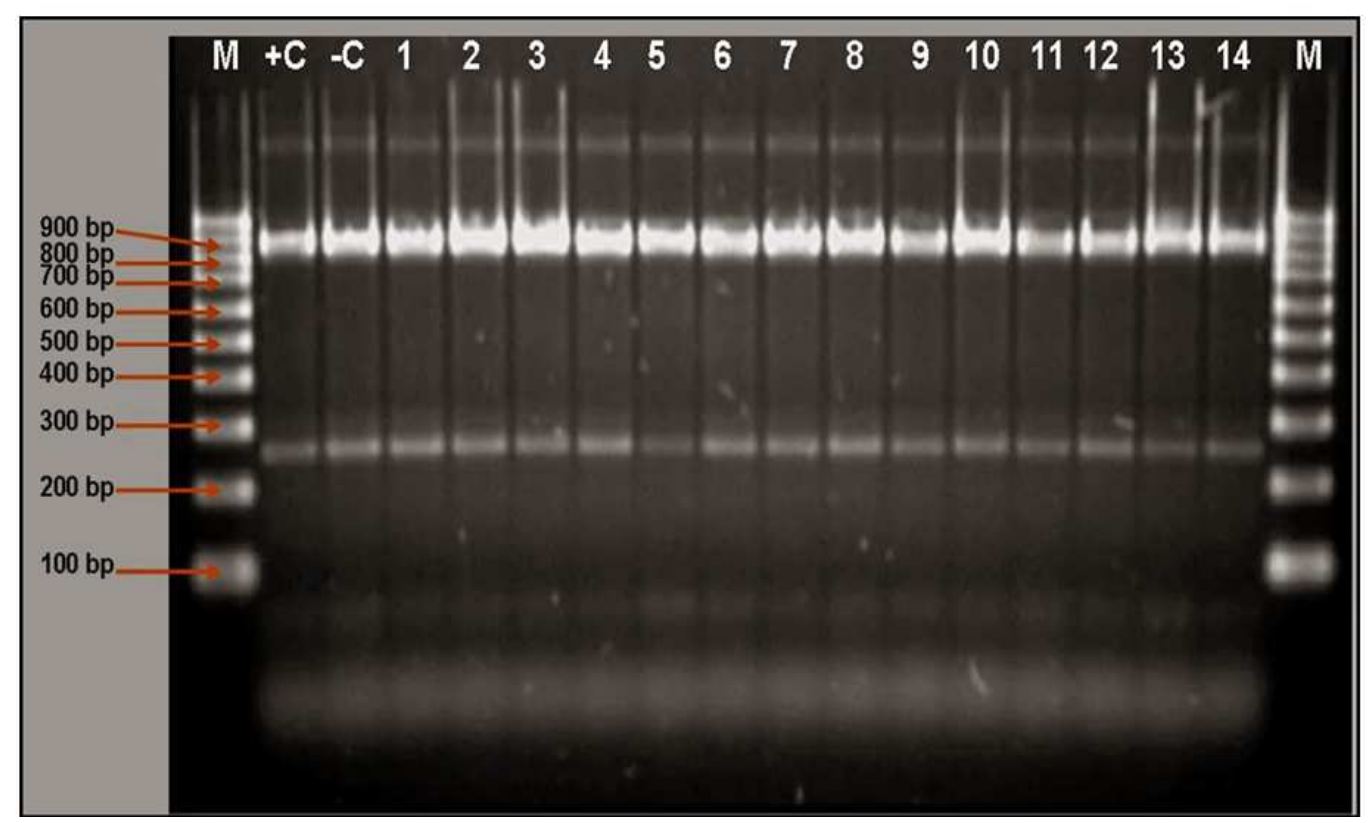

Fig. 6. Interpretation of PCR results after electrophoresis on an agarose gel (1.5\%); Lane M, molecular size marker (MBI Fermentas ladder mix, Germany); Lane +C, S. aureus reference strain ATCC 29213 (positive control); Lane -C, negative control containing water; Lanes 1-14 positive 16S rRNA and nuc genes (Positive S. aureus strains. A strong signal of DNA band was detected in all strains from 1- 14

Usually, the MALDI Biotyper pattern-matching algorithm considers the matches of the unknown sample spectrum against the reference database and the reverse matches of the major spectrum with the unidentified spectrum; it moreover evaluates the relative intensities of nameless and database spectra (Lartigue et al., 2009). As well, MALDI-TOF MS is capable of recognize mixed bacterial cultures, as demonstrated by two of the samples of $S$. aureus that identified as by biochemical testing $S$. epidermidis. The MALDI-TOF MS and conventional identifications were carried out for all 39 $S$. aureus samples. Each sample was cultured twice and similar results were observed. Thirty five out of thirty nine bacterial isolates presented the same results through both identification methods. The MALDI Biotyper Database comprises data from strain collections (e.g., American Type Culture Collection, ATCC) and sequenced bacterial strains and has been optimized for Strept. agalactiae identification (Lartigue et al., 2009).

In the current study, four samples of $S$. aureus were identified as $S$. epidermidis by the biochemical measures previously declared, while two samples of the four isolates documented by MALDI-TOF MS were identified as $S$. aureus. To confirm some identification results provided by MALDI-TOF MS as compared with the classical microbiology results, the $16 \mathrm{~S}$ rRNA and nuc genes of certain samples were sequenced. Consequently, nuc-based PCR detection was utilized as a confirmation method for MALDI-TOF MS analysis in the current study. The results of nuc-based PCR detection demonstrated that all the thirty nine isolates in this study were $S$. aureus (Fig. 6).

\section{DISCUSSION}

One hundred twenty isolates of various mastitis pathogens such as $S$. aureus, CNS and Strept. agalactiae were analyzed by conventional (as API ID System and VITEK 2 System) and Mass Spectral (MALDI-TOF-MS) methods. According to the results, $\sim 95 \%$ of S. aureus, $100 \%$ of CNS and Strept. agalactiae were correctly identified by MALDI TOF MS. All $S$. aureus isolates were then confirmed by a nuc-based PCR technique. While $\sim 92 \%$ of S. aureus, $87 \%$ of Strept. agalactiae and $76 \%$ of CNS were identified by VITEK 2 system. Moreover, $\sim 89 \%$ of S. aureus, $80 \%$ of Strept. agalactiae and $72 \%$ of CNS were identified by API system. The results tabulated in the current study, supported by similar and widespread data for clinical human and veterinary microbiology (Ilina et al., 2009; Lartigue et al., 2009; 
Marklein et al., 2009; Nagy et al., 2009; Barreiro et al., 2010; Dubois et al., 2010; Ilina et al., 2010; Seibold et al., 2010; Moon et al., 2013; Raemy et al., 2013), confirmed that MALDI-TOF MS contributes in the fast identification of bacteria (one day versus five to eight days for conventional identification) and consequently the chance of an earlier treatment of subclinical and clinical mastitis with suitable antimicrobial agents will be carried out. In addition, in the dairy industry, MALDITOF MS can provides a faster, cheaper and more reliable identification of microorganisms isolated from milk of animals witth clinical and subclinical mastitis. As MALDI-TOF MS is an innovative technique that will definitely change the way of functioning of microbiology laboratories, additional future researches aimed at evaluating its cost-effectiveness and time to results in comparison to those for conventional methods will be required.

Previously, identification of microorganisms is usually accomplished using phenotypic based techniques. Nevertheless, Sampimona et al. (2009) indicated that conventional methods such as the API Staph ID 32 and the Staph-Zym had a high number of unidentified or misidentified CNS strains. Both tests were inadequate to identify CNS isolates from mastitis milk samples. In addition, ribotyping and PCR amplicons-sequencing based methods for identification of different types of bacteria still prolonged, expensive and skilled demanding. In contrast, using of MALDITOF-MS technique, sample preparation and analysis are uncomplicated and can be achieved within few minutes. Biswas and Rolain (2013) found that no particular lysis stage is required beyond the exposure to the matrix solution and the device does not require a professional handler. Just a loopful of bacterial colonies is required for analysis by MALDI-TOF-MS and the side view is produced with smallest consumables and cost. For one sample of bacteria, mass spectral identification is achieved in a few minutes versus five to seven days for phenotypical identification. Numerous samples can be achieved per day and moreover the cost of the analysis is cheap compared to other techniques (in the range of a few cents).

The mass spectral data were evaluated with software supplied with the instrument. The bacterial strains in the current research were recognized by comparing their spectral profiles with the database, which is composed of more than three hundred strains of $\sim 60$ genera from National Collection of Type Cultures (NCTC), including eighteen species of genus Staphylococcus. The pattern identification algorithm utilizes all the mass intensity data in the mass spectrum to provide the most outstanding database match (Barreiro et al., 2010) Therefore, 100\% of CNS was accurately recognized by MALDI-TOF MS (50 isolates of CNS yielded strong signals in the mass spectrum, which led to correct identification). In addition, $100 \%$ of thirty one isolates of Strep. agalactiae were accurately identified without any weak signal in the mass spectrum. While $\sim 95 \%$ of 39 isolates of $S$. aureus were properly detected by MALDI-TOF MS (only two strains produced weak signals in the mass spectrum, which led to failure of identification). According to the conditions of the current study, most spectral peaks noticed were in the mass range of $\mathrm{m} / \mathrm{z}$ 2000-18000 Da, Similar ranges were noticed by (Barreiro et al., 2010; Raemy et al., 2013) who stated that the most spectral peaks resulted from analysis of protein samples were ranged from 2000 to 20000 Da mass range. The difference in mass range of spectra peaks might be due to the variation in techniques of sample preparation.

In this study, it is discovered that MALDI-TOF-MS is a powerful technique for the identification of different strains of contagious mastitis pathogens as S. aureus, Strep. agalactiae and CNS isolated from milk of cows affected by clinical and subclinical mastitis. The scheme reported in this study is currently being extended to major types of microorganisms isolated in veterinary and human laboratories of microbiology. Consequently, microbial identification in clinical laboratories depending on two steps. The primary step is a fast detection of the isolated pathogen by standard phenotypic identification such as growth conditions, gram staining and morphology, therefore this step helping in classification of the pathogen within a group of bacteria frequently isolated in clinical microbiology laboratories, e.g., Micrococcaceae, catalase-positive, aerobic gram positive cocci. The second step depends on mass spectral identification by MALDI-TOF-MS, permitting the fast identification of the species. So The current study proposed that an alternative technique may be permitted in the future instead of conventional methods, which are time-consuming and not reliable.

Barreiro et al. (2010) noticed that identification of Strept. uberis isolated from milk samples of cow with subclinical mastitis by standard biochemical reactions were not accurate, time consuming and several errors may be resulted during sample preparation. Approaches at the molecular level have long been known as significant tools in the bacterial identification Cobo, 2013 and MALDITOF MS utilizing "molecular fingerprints" seems to supply a trustworthy tool for identification of different types of microorganisms. This seems to open a novel era 
in microbiology screening in which traditional phenotypic techniques will change to molecular techniques able to providing quicker and more correct responses and similar genome-related methods of taxonomic and phylogenetic analysis (Barreiro et al., 2010).

\section{CONCLUSION}

A successful mangement plan for clinical and subclinical bovine mastitis can be established with an effective monitoring system for all dairy herds and accurate identification of microorganisms that cause mastitis. Mass spectral identification of bacterial cells by MALDI-TOF-MS requires little effort for sample preparation and can be accomplished within a few minutes. In this study, MALDI-TOF-MS is shown to be a more fast and influential tool for the fast identification of different strains of S. aureus, Strep. agalactiae and CNS. Therefore, it is recomended that MALDI-TOF MS technology can be regularly used in veterinary laboratories for identification of different species of bacteria, particularly when failure of phenotypic methods forces clinical microbiologists. Future uses of MALDI-TOF MS in clinical bacteriology will be developed in order to provide more precise identification on clinical specimens and will include the detection of resistance protein markers and specific virulence for several microbes. Nevertheless, the introduction of this technology routinely for microbiology diagnosis would enable a change in working practices. Furthermore, this truth will need a complete integration into laboratory workflow and several technical developments such as to detection sensitivity. The continuous updating of databases for microorganisms will be also a significant issue, in addition the opportunity for the diagnosis directly from clinical samples.

\section{ACKNOWLEDGEMENT}

Appreciation is expressed to all the technical staff of the laboratory Department of Bukayriyah General Hospital, Al-Qassim region, Saudia Arabia for their contribution in the practical part of this study.

\section{REFERENCES}

Alispahic, M., K. Hummel, D. Jandreski-Cvetkovic, K. Nöbauer and E. Razzazi-Fazeli et al., 2010. Speciesspecific identification and differentiation of arcobacter, helicobacter and campylobacter by full-spectral matrixassociated laser desorption/ionization time of flight mass spectrometry analysis. J. Med. Microbiol., 59: 295-301. DOI: 10.1099/jmm.0.016576-0
Barreiro, J.R., C.R. Ferreira, G.B. Sanvido, M. Kostrzewa and T. Maier et al., 2010. Short communication: Identification of subclinical cow mastitis pathogens in milk by matrix-assisted laser desorption/ionization time-of-flight mass spectrometry. J. Dairy Sci., 93: 5661-5667. DOI: 10.3168/jds.2010-3614

Benić, M., H. Boris and G. Kompes, 2012. Clinical and epidemiological aspects of cow mastitis caused by Staphylococcus aureus and its methicillin-resistant strains. J. Med. Sci., 37: 113-121.

Biswas, S. and J.M. Rolain, 2013. Use of MALDI-TOF mass spectrometry for identification of bacteria that are difficult to culture. J. Microbiol. Meth., 92: 1424. DOI: $10.1016 /$ j.mimet.2012.10.014

Boss, R., J. Naskova, A. Steiner and H.U. Graber, 2011. Mastitis diagnostics: quantitative PCR for Staphylococcus aureus genotype B in bulk tank milk. J. Dairy Sci., 94: 128-137. DOI: 10.3168/jds.2010-3251

Castelani, L., A.F. Santos, M.D.S. Miranda, L.F. Zafalon and C.R.C.R. Pozzi et al., 2013. Molecular typing of mastitis-causing staphylococcus aureus isolated from heifers and cows. Int. J. Mol. Sci., 14: 43264333. DOI: $10.3390 /$ ijms 14024326

Chalupová, J., M. Raus, M. Sedlářovám and M. Sebela, 2014. Identification of fungal microorganisms by MALDI-TOF mass spectrometry. Biotechnol Adv., 32: 230-41. DOI: 10.1016/j.biotechadv.2013.11.002

Dubois, D., D. Leyssene, J.P. Chacornac, M. Kostrzewa and P.O. Schmit et al., 2010. Identification of a variety of Staphylococcus species by matrix-assisted laser desorption ionization-time of flight mass spectrometry. J. Clin. Microbiol., 48: 941-945. DOI: 10.1128/JCM.00413-09

Dudko, P., K. Kostro and M. Kurpisz, 2010. Adaptation of microstix ${ }^{\circledR}$ candida slide-test for diagnosis of bovine mastitis due to anascogenic yeasts. ACTA VET. BRNO, 79: 113-120. DOI: 10.2754/avb201079010113

El-Bouri, K., S. Johnston, E. Rees, I. Thomas and N. BomeMannathoko et al., 2012. Comparison of bacterial identification by MALDI-TOF mass spectrometry and conventional diagnostic microbiology methods: Agreement, speed and cost implications. Br. J. Biomed. Sci., 69: 47-55. PMID: 22872927

Giebel, R., C. Worden, S.M. Rust, G.T. Kleinheinz and M. Robbins et al., 2010. Microbial fingerprinting using Matrix-Assisted Laser Desorption Ionization Time-Of-Flight Mass Spectrometry (MALDI-TOF MS) applications and challenges. Adv. Appl. Microbiol., 71: 149-184. DOI: 10.1016/S00652164(10)71006-6 
Hays, J.P. and W.B.V. Leeuwen, 2012. The Role of New Technologies in Medical Microbiological Research and Diagnosis. 1st Edn., Bentham Science Publishers, Sharjah, ISBN-10: 1608053164, pp: 170.

Ilina, E.N., A.D. Borovskaya, M.M. Malakhova, V.A. Vereshchagin and A.A. Kubanova et al., 2009. Direct bacterial profiling by matrix-assisted laser desorptionionization time-of-flight mass spectrometry for identification of pathogenic Neisseria. J. Mol. Diagn., 11: 75-86. DOI: 10.2353/jmoldx.2009.080079

Ilina, E.N., A.D. Borovskaya, M.V. Serebryakova, V.V. Chelysheva and K.T. Momynaliev et al., 2010. Application of matrix-assisted laser desorption/ionization time-of-flight mass spectrometry for the study of Helicobacter pylori. Rapid Commun. Mass Spectrom., 24: 328-334. DOI: 10.1002/rcm.4394

Johler, S., P.S. Tichaczek-Dischinger, J. Rau, H.M. Sihto and A. Lehner et al., 2013. Outbreak of Staphylococcal food poisoning due to SEA-producing Staphylococcus aureus. Foodborne Pathogen and Disease, 10: 777-781. DOI: 10.1089/fpd.2013.1503

Karger, A., R. Stock, M. Ziller, M.C. Elschner and B. Bettin et al., 2012. Rapid identification of Burkholderia mallei and Burkholderia pseudomallei by intact cell Matrix-assisted Laser Desorption/Ionisation mass spectrometric typing. BMC Microbiol., 12: 229244. DOI: $10.1186 / 1471-2180-12-229$

Koskinen, M.T. G.J. Wellenberg, O.C. Sampimon, J. Holopainen and A. Rothkamp et al., 2010. Field comparison of real-time polymerase chain reaction and bacterial culture for identification of bovine mastitis bacteria. J. Dairy Sci., 93: 5707-5715. DOI: 10.3168/jds.2010-3167

Lartigue, M.F., G. Héry-Arnaud, E. Haguenoer, A.S. Domelier and P.O. Schmit et al., 2009. Identification of Streptococcus agalactiae isolates from various phylogenetic lineages by matrixassisted laser desorption ionization-time of flight mass spectrometry. J. Clin. Microbiol., 47: 22842287. DOI: 10.1128/JCM.00175-09

Marklein, G., M. Josten, U. Klanke, E. Müller and R. Horré et al., 2009. Matrix-assisted laser desorption ionization-time of flight mass spectrometry for fast and reliable identification of clinical yeast isolates. J. Clin. Microbiol., 47: 2912-2917. DOI: 10.1128/JCM.00389-09

Mellmann, A., F. Bimet, C. Bizet, A.D. Borovskaya and R.R. Drake et al., 2009. High inter-laboratory reproducibility of matrix-assisted laser desorption ionization-time of flight mass spectrometry-based species identification of non-fermenting bacteria. J. Clin. Microbiol., 47: 3732-3734. DOI: 10.1128/JCM.00921-09
Moon, H.W., S.H. Lee, H.S. Chung, M. Lee and K. Lee, 2013. Performance of the Vitek MS matrix-assisted laser desorption ionization time-of-flight mass spectrometry system for identification of Grampositive cocci routinely isolated in clinical microbiology laboratories. J Med Microbiol., 62: 1301-1306. DOI: 10.1099/jmm.0.062950-0

Nagy, K., K. Redeuil, R. Bertholet, H. Steiling and M. Kussmann, 2009. Quantification of anthocyanins and flavonols in milk-based food products by ultra performance liquid chromatography-tandem mass spectrometry. Anal. Chem., 81: 6347-6356. DOI: 10.1021/ac900608g

Pradhan, P., S.M. Gopinath, G.R. Reddy, H.J. Dechamma and V.V.S. Suryanarayana, 2011. Detection of major pathogens in bovine sub-clinical mastitis by multiplex PCR directly from milk samples in presence of an internal control. I. J. 1: 209-218

Raemy, A., M. Meylan, S. Casati, V. Gaia and B. Berchtold et al., 2013. Phenotypic and genotypic identification of streptococci and related bacteria isolated from bovine intramammary infections. Acta Vet. Scand., 55: 53-53. DOI: 10.1186/1751-0147-55-53

Saidi, R., D. Khelef and R. Kaidi, 2013. Subclinical mastitis in cattle in Algeria: Frequency of occurrence and bacteriological isolates. J. S Afr. Vet. Assoc., 84: 1-5. DOI: 10.4102/jsava.v84i1.929

Sampimona, O.C., R.N. Zadoksb, S. De Vliegherc, K. Supréc and F. Haesebrouckd et al., 2009. Performance of API Staph ID 32 and Staph-Zym for identification of coagulase negative staphylococci isolated from bovine milk samples. Vet. Microb., 136: 300-305. DOI: 10.1016/j.vetmic.2008.11.004

Seibold, E., T. Maier, M. Kostrzewa, E. Zeman and W. Splettstoesser, 2010. Identification of Francisella tularensis by whole-cell matrix-assisted laser desorption ionization-time of flight mass spectrometry: Fast, reliable, robust and costeffective differentiation on species and subspecies levels. J. Clin. Microbiol., 48: 1061-1069. DOI: 10.1128/JCM.01953-09

Tomazia, T., J.L. Gonçalvesa, J.R. Barreiroa, P.A. de Campos Bragab and L.F. Prada e Silvaa et al., 2014. Identification of coagulase-negative staphylococci from bovine intramammary infection by matrixassisted laser desorption ionization-time of flight mass spectrometry. J. Clin. Microbiol., 52: 16581663. DOI: 10.1128/JCM.03032-13 